\title{
Erratum to: Global standards for global health in a globalized economy!
}

\author{
Nino Künzli $\cdot$ Meltem Kutlar Joss $\cdot$ Emily Gintowt
}

Published online: 22 October 2015

(c) Swiss School of Public Health (SSPH+) 2015

\section{Erratum to: Int J Public Health \\ DOI 10.1007/s00038-015-0729-0}

Unfortunately, the table shows the past rather than the current long-term air quality standards for the USA. As of 2015, the US has no federal annual mean standard for PM10 whereas the PM2.5 standard has been set at $12 \mu \mathrm{g} / \mathrm{m}^{3}$.

China has partially set stricter long-term targets for PM in larger cities which will be valid nationwide by 2016 $\left(70 \mu \mathrm{g} \mathrm{PM} 10 / \mathrm{m}^{3}\right.$ and $\left.35 \mu \mathrm{g} \mathrm{PM} 2.5 / \mathrm{m}^{3}\right)$.

\begin{tabular}{lll}
\hline Country or agency & $\mathrm{PM}_{10} \mu \mathrm{g} \mathrm{m}^{-3}$ & $\mathrm{PM}_{2.5} \mu \mathrm{g} \mathrm{m}^{-3}$ \\
\hline Reference: WHO & $\mathbf{2 0}$ & $\mathbf{1 0}$ \\
Africa & & \\
$\quad$ Kenya & 50 & 35 \\
$\quad$ South Africa & 50 & 25 \\
$\quad$ Tanzania & $60-90$ & $\mathrm{~N} / \mathrm{A}$ \\
Central America & & \\
$\quad$ Costa Rica & 50 & $\mathrm{~N} / \mathrm{A}$ \\
El Salvador & 50 & 15 \\
Jamaica & 50 & $\mathrm{~N} / \mathrm{A}$ \\
Nicaragua & 50 & $\mathrm{~N} / \mathrm{A}$ \\
Panama & 50 & $\mathrm{~N} / \mathrm{A}$ \\
Dominican Republic & 50 & 15 \\
\hline
\end{tabular}

The online version of the original article can be found under doi:10. 1007/s00038-015-0729-0.

N. Künzli $(\square) \cdot$ M. Kutlar Joss · E. Gintowt Swiss Tropical and Public Health Institute, Basel Switzerland and University of Basel, Socinstrasse 57, P.O. Box, 4002 Basel, Switzerland

e-mail: nino.kuenzli@unibas.ch

\begin{tabular}{|c|c|c|}
\hline Country or agency & $\mathrm{PM}_{10} \mu \mathrm{g} \mathrm{m}^{-3}$ & $\mathrm{PM}_{2.5} \mu \mathrm{g} \mathrm{m}^{-3}$ \\
\hline \multicolumn{3}{|l|}{ North America } \\
\hline Canada & N/A & 10 \\
\hline$>$ British Columbia & N/A & 10 \\
\hline Mexico & 40 & 12 \\
\hline Puerto Rico & N/A & 15 \\
\hline USA & N/A & 12 \\
\hline$>$ California & 20 & 12 \\
\hline \multicolumn{3}{|l|}{ South America } \\
\hline$>$ Buenos Aires & 50 & 15 \\
\hline Bolivia & 50 & N/A \\
\hline$>\mathrm{La} \mathrm{Paz}$ & 20 & 10 \\
\hline Brazil & 50 & N/A \\
\hline Chile & 50 & 20 \\
\hline Colombia & 50 & 25 \\
\hline Ecuador & 50 & 15 \\
\hline Peru & 50 & N/A \\
\hline Venezuela & 50 & N/A \\
\hline \multicolumn{3}{|l|}{ Asia/Oceania } \\
\hline Australia & N/A & 8 \\
\hline Bangladesh & 50 & 15 \\
\hline China (residential areas) & 70 & 35 \\
\hline Hong Kong SAR & 55 & N/A \\
\hline India & 60 & 40 \\
\hline Iran & 20 & 10 \\
\hline Israel & 50 & 25 \\
\hline Japan & N/A & 15 \\
\hline Malaysia & 50 & N/A \\
\hline Philippines & 60 & N/A \\
\hline Republic of Korea & 50 & N/A \\
\hline Singapore & N/A & 15 \\
\hline South Korea & 50 & 25 \\
\hline Sri Lanka & 50 & 25 \\
\hline
\end{tabular}




\begin{tabular}{lll}
\hline Country or agency & $\mathrm{PM}_{10} \mu \mathrm{g} \mathrm{m}^{-3}$ & $\mathrm{PM}_{2.5} \mu \mathrm{g} \mathrm{m}^{-3}$ \\
\hline Taiwan & 65 & N/A \\
Thailand & 50 & 25 \\
Uzbekistan & 50 & $\mathrm{~N} / \mathrm{A}$ \\
Vietnam & 50 & 25 \\
Europe & & \\
EU & 40 & 25 \\
Switzerland & $\mathbf{2 0}$ & $(10$ under consideration $)$ \\
Turkey & 60 & N/A \\
\hline
\end{tabular}

Standards set in bold are within $20 \%$ of the science-based values asproposed by WHO to protect public health

N/A or countries not listed: no policies could be identified. For other national air quality standards and for references to the National policies: see the website of Ludok at the Swiss Tropical and Public Health Institute (http://ludok.swisstph.ch)—“Grenzwerte" 